\title{
Magnetic susceptibility measurements of LHP and LNP crystals
}

\author{
C C DESAI and M S V RAMANA \\ Department of Physics, Sardar Patel University, Vallabh Vidyanagar 388 120, India \\ MS received 5 February 1987; revised 6 June 1987
}

\begin{abstract}
Ferroelectric single crystals such as lead hydrogen phosphate (LHP) and dihydrated lead nitrate phosphate (LNP) have been derived from the diffusion of lead nitrate into the set gel containing orthophosphoric acid. By employing Faraday's Gouy balance technique, the magnetic susceptibilities of LHP and LNP have been determined. It has been ohserved that (i) the gram and molar susceptibilitics are independent of magnetic field, (ii) $\gamma_{M}$ of LNP materials are greater than that of LHP materials and (iii) the positive values of magnetic susceptibilities suggest that LHP and LNP are paramagnetic materials.
\end{abstract}

Keywords. Silica gels; ferroelectric; Gouy balänce; magnetic susceptibility; lead hydrogen phosphate; lead nitrate phosphate.

\section{Introduction}

The study of magnetic susceptibility at different magnetic fields is an important tool to probe into the magnetic properties of materials. Considerable amount of work along these lines has been carried out on a variety of materials, e.g. scheelite compound (Godbole 1982) and tin iodates (Desai and Rai 1983) yielding valuable information. LHP and LNP crystals are of considerable interest, particularly in basic studies of their phase transitions and of their ferroelectric, optical and other pertinent characteristics. The information on magnetic properties of ferroelectric crystals, such as LHP and LNP is meagre. In this paper, we report magnetic susceptibilities of these crystals using the Faraday Gouy balance technique.

\section{Experimental}

Single crystals of LHP and LNP were grown using the controlled reaction between lead nitrate and orthophosphoric acid solutions. The crystals were examined by EDAX, X-ray diffraction and density measurements; these techniques confirmed that they were LHP (monoclinic, space group $P 2 / a$ ) and LNP (monoclinic, space group $\left.P 2_{1} / c\right)$.

The magnetic susceptibility of these crystals was measured using the Faraday method (Bates 1951). The finely powdered crystals of LHP and LNP were packed in a Gouy tube of known magnetic susceptibility. This tube was hung between two powerful electromagnetic pole pieces of a Gouy balance whose accuracy was $10^{-5} \mathrm{~g}$. Initially, the samples were weighted accurately in the absence of the magnetic field. The magnetic field was increased by increasing the current from 3 to 7 amp through the coils and the corresponding magnetic field strength was measured with a Gauss meter. The change in weight, i.e. reduced mass of samples, after applying the magnetic field was measured. 
The positive gram susceptibility, $\chi_{g}$, characteristic of a paramagnetic material was determined using the formula

$$
\chi_{g}=(\alpha+\beta d w) / w
$$

where $\alpha$ is a constant which represents the correction factor due to displacement of air, $\beta$ the constant for a glass container at a given current, $w$ the weight of the sample, $d w$ the change in weight when a magnetic field is applied. The gram susceptibility of the material was multiplied by its molecular mass to obtain molar susceptibility $\left(\chi_{M}\right)$.

\section{Results and discussion}

LHP and LNP grown crystals are usually transparent, white in colour and are rectangular in shape. These crystals are finely ground and the resulting powder (particle size $150 \mu \mathrm{m}$ ) were packed in a Gouy tube of known magnetic susceptibility. The experiments on magnetic susceptibility measurements were repeated five times and the average change in weight was computed for a given current. The accuracy in the measurement of reduced mass was $\pm 5 \%$. The readings of the Gouy balance were recorded when the values became steady. The values were quite consistent and reproducible.

As the magnetic field increases, the change in weight, i.e. the reduced weight also increases. The plot of magnetic susceptibility, $\chi$ against magnetic field $(H)$ is illustrated in figure 1. From this figure it is clear that (i) when magnetic field is applied the reduced weight of all samples is increased, (ii) the magnetic susceptibilities $\left(\chi_{g}\right.$ and $\chi_{M}$ ) are independent of the current, (iii) $\chi_{M}$ of LNP samples are greater than that of LHP samples, and (iv) the positive values of the susceptibilities thereby suggest that LHP and LNP are paramagnetic materials. This may be attributed to the presence of lead (unfilled $p$ shells) in them.

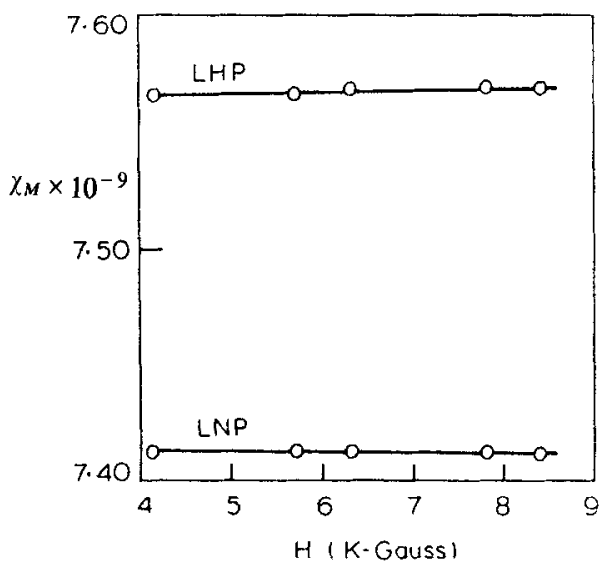

Figure 1. Plots of magnetic susceptibility $\left(\chi_{M}\right)$ against magnetic field $(H)$ for LHP and LNP crystals. 


\section{Acknowledgements}

The authors thank CSIR, New Delhi for financial assistance. One of us (CCD) is grateful to Mrs Gangaba Desai for her keen interest.

\section{References}

Bates L F 1957 Modern magnetism (Cambridge: University Press) pp 101-109

Desai C C and Rai J L 1983 J. Mater. Sci. Lett. 2467

Godbole R S 1982 Growth and characterization of tungstate group crystals, Ph.D. thesis, Sardar Patel University, Vallabh Vidyanagar 